\title{
Generic nonequilibrium steady states in an exclusion process on an inhomogeneous ring
}

\author{
Tirthankar Banerjee,, , 因 Niladri Sarkar, ${ }^{1,}$ 团 and Abhik Basu ${ }^{1}$, 团 \\ ${ }^{1}$ Condensed Matter Physics Division, Saha Institute of Nuclear Physics, Calcutta 700064, India
}

(Dated: June 4, 2018)

\begin{abstract}
We consider a one-dimensional totally asymmetric exclusion process on a ring with extended inhomogeneities, consisting of several segments with different hopping rates. Depending upon the underlying inhomogeneity configurations and for moderate densities, our model displays both localised (LDW) and delocalised (DDW) domain walls and delocalisation transitions of LDWs in the steady states. Our results allow us to construct the possible steady state density profiles for arbitrary number of segments with unequal hopping rates. We explore the scaling properties of the fluctuations of LDWs and DDWs.
\end{abstract}

\section{INTRODUCTION}

Totally asymmetric simple exclusion process (TASEP) in one dimension (1D) serves as a paradigmatic model for systems out of equilibrium, where fundamental questions concerning nonequilibrium statistical mechanics can be asked and explicitly answered. Phenomenologically, it is used as a simple physical description for a variety of systems ranging from quasi-1D transport in cell biology contexts, e.g., ribosome translocations along messenger RNA (mRNA) [1] to fluid motion along artificial crystalline zeolitical structures [2] and movement of unidirectional vehicular traffic along roads [3]. The novelty of TASEP as an open system lies in its ability to display boundary-induced phase transitions [4]. Several different variants of TASEP [5, 6], including effects of inhomogeneities have been studied [7, 8], shedding insight for nonequilibrium physics.

In this article, we consider the steady state densities in TASEP in an inhomogeneous system with periodic boundary conditions, e.g., a closed ring. Unlike open systems, closed-ring systems admit strict particle number conservation, which is known to affect the universal scaling behavior near a critical point in uniform systems [9]. However, TASEP on a uniform ring cannot display spatially non-trivial steady state density profiles, due to the spatial translation invariance of the model. Only when translational invariance is broken explicitly, e.g., by connections with diffusive lanes, presence of defects or appearance of junctions and branches, inhomogeneous density profiles may appear $[10-12]$. In general, steady state properties of asymmetric exclusion processes on a ring with inhomogeneities have been studied by using various models. For instance, Ref. [12] considered TASEP on a ring with one point defect (i.e., a single slow site) and found LDWs in the steady states under certain conditions. Subsequently, Ref. [1] extensively

\footnotetext{
*Electronic address: tirthankar.banerjee@saha.ac.in

${ }^{\dagger}$ Electronic address: niladri.sarkar@saha.ac.in

‡Electronic address: abhik.basu@saha.ac.in
}

discussed disordered asymmetric exclusion processes by using the fully segregated model (FSM), where the system has two parts with different hopping rates; see also Refs. 13, 14 for detailed studies on the various aspects of the effects of quenched disorders on TASEP, Ref. 15] discussed the effects of quenched disorders on TASEP and their connections with the dynamic scaling of KardarParisi-Zhang [16] surfaces.

In the present work, we are generally motivated to study the possible density profiles with finite number of segments having unequal hopping rates as consequences of the mutual interplay between conservation laws and the scale of inhomogeneities in closed exclusion processes. We extend and complement the existing studies on closed TASEP with inhomogeneities by considering a model of exclusion process on a closed inhomogeneous ring having multiple segments with unequal hopping rates. Our model reveals nonequilibrium phase transitions including delocalisation transitions of LDWs into delocalised domain walls (DDWs), distinctly different from boundary induced phase transitions in an open TASEP (see, e.g., Ref. 17] for boundary induced phase transitions in driven diffusive systems). We discuss how these may be generalised for larger number of segments. In addition, we numerically explore the nature of fluctuations of LDWs and DDWs in the system and the associated delocalisation transition. Our results should be of significance in understanding the phenomenologies of ribosome translocations along closed mRNA loops (circular translation of polysomal mRNA) [18, 19] with clusters of slow codons along which ribosome translocations are inhibited [20 22]. We use analytical mean-field theories (MFT) complemented by extensive Monte Carlo simulation (MCS) studies for our work. Our main findings in this article are as follows: (i) When there are three or four segments of all unequal hopping rates, there should be one LDW in the system with the slowest segment being in the maximal current (MC) phase for moderate densities, (ii) for moderate densities with four segments having two non consecutive slowest segments (of equal hopping rates), instead of a single LDW, there will be two DDWs, each formed behind one of the slowest segments; the slowest segments themselves are in their MC phases, (iii) we use 
our results from the three- and four-segment models to infer about the possible general forms of the steady state density profiles for arbitrary number of segments with different hopping rates, and (iv) we numerically uncover the scaling properties of widths of the LDW fluctuations, both near and away from delocalisation transitions. The remaining parts of this paper are organised as follows.In Sec. III we present a brief review of the FSM. In Secs. III and [V] we construct our models with three (Model I) and four (Model II) segments, and study in details the corresponding steady state density profiles. In Sec. V] we use our results for three and four segments to comment on systems with arbitrary number of segments. Lastly, in Sec. VI, we investigate the scaling of the fluctuations of LDW and DDWs in our MCS studies. In Sec. VII we summarise and conclude.

\section{REVIEW OF THE TWO-CHANNEL SYSTEM}

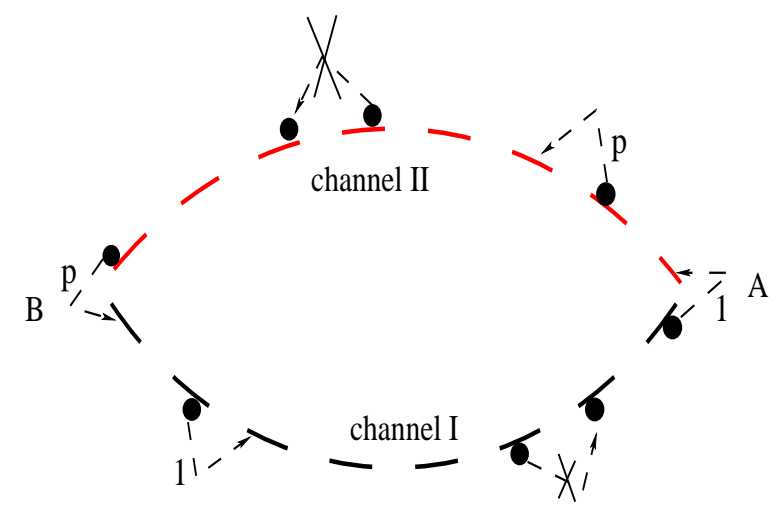

FIG. 1: Schematic diagram of the FSM model. CHI (black) has a hopping rate 1 , CHII (red) has a hopping rate of $p<1$ (see text).

In order to set up the background, it is instructive to first review the results from the analogous two-segment system (FSM), as elucidated in Ref. [11]. It has two parts, $\mathrm{CHI}$ and $\mathrm{CHII}$, of hopping rate 1 and $p$, respectively; see Fig. 1 for a schematic diagram. Assume equal lengths for $\mathrm{CHI}$ and CHII. If we represent a phase of the whole system by $(X, Y)$, then $(X, Y)$ can be any of low density (LD), high density (HD), domain wall or co-existence (DW) and maximal current (MC) phase of $\mathrm{CHI}$ and CHII, respectively. As discussed in Ref. [11] in great details, the system can be only in three out of all possible sixteen phases. These three phases are (LD,LD), (HD,HD) and (DW,MC).

The above physical picture [11] can be made quantitative as follows. Consider first the (LD,LD) or (HD,HD) phases and ignore the boundary layers (BLs) (having vanishing width in thermodynamic limit) in MFT, for which the bulk densities $n_{I}$ and $n_{I I}$ are spatially constants in the steady states. Then, $J_{I}=J_{I I}$ in the steady states gives

$$
n_{I}\left(1-n_{I}\right)=p n_{I I}\left(1-n_{I I}\right)
$$

With the particle number density given by

$$
n=\frac{n_{I}+n_{I I}}{2}
$$

we obtain

$$
n_{I I}=\frac{-b \pm \sqrt{b^{2}-4 a c}}{2 a}
$$

with $a=(p-1) / 4, b=n-\frac{(1+p)}{4}$ and $c=n / 2-n^{2}$. These yield densities $n_{I I}$ and $n_{I}$ when both of them are in their LD or HD phases. The two thresholds for $n$, namely, $n_{L}, n_{U}$, which mark the boundaries between LD/MC or $\mathrm{HD} / \mathrm{MC}$ phases for CHII (or, equivalently, the boundaries between $\mathrm{LD} / \mathrm{DW}$ or $\mathrm{HD} / \mathrm{DW}$ phases for $\mathrm{CHI}$ ) may be obtained by setting $n_{I I}=1 / 2$ (see below).

Consider CHII to be in its MC phase, while CHI is to have a coexistence of LD and HD phases in the form of a DW or a discontinuity in its density profile. By using $J_{I}=J_{I I}$, we get

$$
n_{I}^{2}-n_{I}+\frac{p}{4}=0
$$

This gives us the solutions $n_{I}=\frac{1 \pm \sqrt{1-p}}{2}$, both corresponding to the same $J_{I}$. The two solutions meet at a point $x_{I}$ in the form of a DW in CHI. More specifically, since CHII has a hopping rate $p$, lower than the hopping rate (unity) in $\mathrm{CHI}$, on simple physical ground we expect particles to pile up behind the junction $\mathrm{B}$, so that when there is a discontinuity in $n_{I}(x): n_{I}=(1-\sqrt{1-p}) / 2<$ $1 / 2$ starting from $x=0$, then jumps discontinuously at $x_{I}\left(0<x_{I}<1-l\right)$ to $n_{I}=(1+\sqrt{1-p}) / 2>1 / 2$ up to $x=1-l$. Thus, in terms of the standard notations of a TASEP with open boundaries, we identify the particle injection rate $\alpha=(1-\sqrt{1-p}) / 2<1 / 2$ and particle extraction rate $\beta=(1-\sqrt{1-p}) / 2<1 / 2$, thus giving $\alpha=\beta<1 / 2$, in agreement with the condition for coexistence in open TASEP. With a DW at $x_{I}$, we have

$$
n_{I}=\alpha_{I}+\theta\left(x-x_{I}\right)\left(1-\alpha_{I}-\beta_{I}\right)
$$

$\theta\left(x-x_{I}\right)$ is the Heaviside step function. Hence, there is a jump in density by a value $1-\beta_{I}-\alpha_{I}$ at $x=x_{I}$ giving the height of the DW. Using particle number conservation $\left(\int_{0}^{1-l} n_{I}+\int_{1-l}^{1} n_{I I}\right) d x=n$, we find

$$
x_{I}=-\frac{n-\left(\frac{\sqrt{1-p}}{2}\right)\left(\frac{1}{2}\right)-\frac{1}{2}}{\sqrt{1-p}} .
$$

The phase boundaries (values for $n_{L}$ and $n_{U}$ ) are obtained by setting $x_{I}=0$ and $x_{I}=1 / 2$ (see Ref. [11] for a phase diagram). 


\section{MODEL I: THREE SEGMENT SYSTEM}

We consider a closed 1D ring having total $N$ sites with three parts of equal lengths but with different hopping rates (henceforth Model I). Equivalently, our model can be viewed as a TASEP on a ring with extended inhomogeneities or defects; see Fig. 2 for a schematic diagram. The three parts, marked CHI, CHII and CHIII (in Fig. 2) are equal in length having $N / 3$ sites each. Segments CHI, CHII and CHIII, respectively have hopping rates unity (with sites $i=1, \ldots, N / 3$ ), $p<1$ (with sites $i=N / 3+1,2 N / 3$ ) and $q<p<1$ (with sites $i=2 N / 3+1, \ldots, N)$. Particle exchanges between each pair of channels are exclusively allowed at their junctions $\mathrm{A}, \mathrm{B}$ and $\mathrm{C}$ with dynamic rules defined by the originating site. If $n_{I i}, n_{I I i}$ and $n_{I I I i}$ represent the average number denisities in $i$-th sites of CHI, CHII and CHIII, respectively and $N_{t o t}$ denotes the total number of particles in the system, we can write,

$$
\left(n_{I}+n_{I I}+n_{I I I}\right) \frac{N}{3}=N_{t o t},
$$

where, $n_{I}=\sum_{i} n_{I i}$ (similarly for $n_{I I}$ and $n_{I I I}$ ). The mean number density for the total system, $n$ can thus be defined as

$$
n=\frac{N_{t o t}}{N}=\frac{n_{I}+n_{I I}+n_{I I I}}{3} .
$$

We are, thus, left with a three-dimensional parameter space spanned by $n, p$ and $q$, each of which is a bulk quantity and confined between 0 and 1 . Tuning the phase parameters, we obtain the various possible phases of the system.

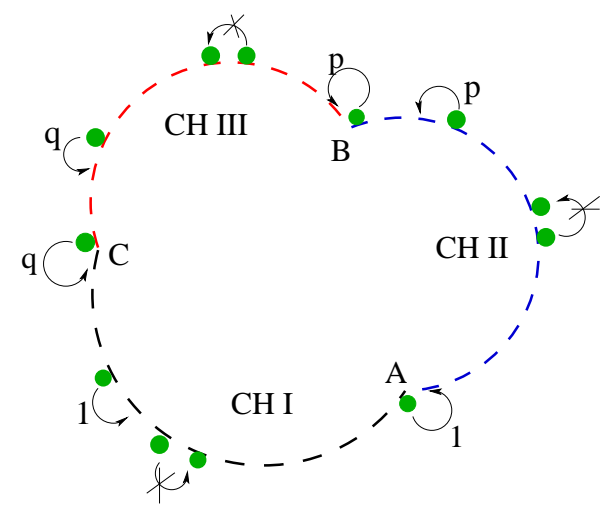

FIG. 2: Schematic diagram of the 3-sector model. CHI (black) has a hopping rate 1 , CHII (blue) has a hopping rate of $p<1$ and CHIII (red) has a hopping rate $q<p$ (see text).

\section{A. MF analysis of Model I}

We use and extend the idea outlined above. In our $\mathrm{MF}$ analysis we treat $\mathrm{CHI}, \mathrm{CHII}$ and $\mathrm{CHIII}$ as separate
TASEP lanes joined at junctions A, B and C, and analyse the phases of the model in terms of the known phases of an open-boundary TASEP. For convenience, we label the sites by a continuous variable $x$ in the thermodynamic limit, defined by $x=i / N, 0<x<1$. We represent a phase of the whole system by $(X, Y, Z)$, where $X, Y, Z$ can be either the LD, HD, MC or DW phases of CHI,CHII and CHIII, respectively. Thus, all in all, there can be at most $4 \times 4 \times 4=64$ phases in the whole system. We now discuss whether or not all the phases are allowed by the constraints of the system. With the bulk densities $n_{I}, n_{I I} n_{I I I}$ respectively, in CHI, CHII and CHIII, the corresponding steady state currents are given by $J_{I}=$ $n_{I}\left(1-n_{I}\right), J_{I I}=p n_{I I}\left(1-n_{I I}\right)$ and $J_{I I I}=q n_{I I I}(1-$ $\left.n_{I I I}\right)$, with $J_{I}=J_{I I}=J_{I I I}$ in the steady states.

Consider the case where the particle motion is from CHI to CHIII via CHII. We argue now that phases (LD,LD,HD),(LD,HD,HD) and (LD,HD,LD) are ruled out. We use the following characterisation of the phases in a TASEP with open boundaries to delineate the phases in CHI, CHII and CHIII of our model:

- When a segment CHX (X= I, II, III) is in its low density (i.e., $n_{X}<0.5$ ), there is no BL in the vicinity of the entrance of CHX. We denote the corresponding uniform steady state in the bulk by $\alpha_{X}$, which is same as the injection rate.

- When a segment CHX (X= I, II, III) is in its high density (i.e., $n_{X}>0.5$ ), there is no $\mathrm{BL}$ in the vicinity of the exit end of CHX. We denote the corresponding uniform steady state in the bulk by $1-\beta_{X}$, where $\beta_{X}$ is the corresponding extraction rate.

For concreteness, consider first the possibility of the (LD,LD,HD) phase, with $\alpha_{I}, \alpha_{I I}$ and $1-\beta_{I I I}$ being the bulk densities of CHI, CHII and CHIII, respectively. Accordingly, the junction marked $C$ in Fig. 2 has no BL, where as the junction $B$ will have two BLs on both sides. We now use current conservation across junction $C$ and in the bulk of CHI and CHIII. These yield

$$
q\left(1-\beta_{I I I}\right) \beta_{I I I}=q \beta_{I I I}\left(1-\alpha_{I}\right)=\alpha_{I}\left(1-\alpha_{I}\right) .
$$

Thus, we must have (i) $1-\beta_{I I I}=1-\alpha_{I}$ and (ii) $q \beta_{I I I}=\alpha_{I}$. Therefore, the only consistent solution of the above is $q=1$, making CHI and CHIII identical. Thus, if $q \neq 1$, there must be a BL at $C$, which in turn rules out the (LD,LD,HD) phase. Similarly the (LD,HD,HD) and (LD,HD,LD) phases also do not appear. Using the particle-hole symmetry [4], the (HD,HD,LD),(HD,LD,LD) and (HD,LD,HD) phases are also ruled out immediately. Furthermore, CHI and CHII can never be in their respective MC phases (corresponding to a currents $1 / 4$ and $p / 4$, respectively), since, the maximum permissible current in the system (with $q \neq 1, p)$ is just $q / 4<1 / 4, p / 4$. Hence, the system has no phases of the form $(\mathrm{MC}, Y, Z)$ or $(Y, \mathrm{MC}, Z)$ where 
$Y, Z$ can be any of $\mathrm{LD}, \mathrm{HD}, \mathrm{MC}$ or DW phases. Additionally, we can make the following general observations concerning the nature of allowed phases in the system. With $J_{I}=J_{I I}=J_{I I I}$ in the steady states, for each of CHI, CHII and CHIII in the LD (HD) phases, $n_{I}<(>) n_{I I}<(>) n_{I I I}$. For very low $n$, all the channels are to be in their respective LD phases, with the bulk densities $n_{I I I}>n_{I I}>n_{I}$. Thus, with increasing $n, n_{I I I}$ reaches a value $1 / 2$, at which CHIII enters its MC phase, with $n_{I}$ and $n_{I I}$ each still less than $1 / 2$. Using the particle-hole symmetry we draw the (equivalent) physical picture of the borderline between the $\mathrm{MC}$ and HD phases of CHIII with $n_{I}, n_{I I}>1 / 2$. Hence, on physical grounds, we identify different thresholds, $n_{L}$ and $n_{U}$, for $n$, that demarcate the LD and MC phases, and MC and HD phases of CHIII, respectively. The values for $n_{L}$ and $n_{U}$ are obtained below. Clearly, with $n_{L}<n<n_{U}$, CHIII remains in its $\mathrm{MC}$ phase, i.e., $n_{I I I}=1 / 2$ (neglecting the BLs in CHII) corresponding to a steady state current $q / 4$ in the system. Therefore, as $n$ rises from $n_{L}$ to $n_{U}$, all the added particles must accumulate in $\mathrm{CHI}$ and CHII, such that $J_{I}=J_{I I}=J_{I I I}=q / 4$ is maintained. This suggests a coexistence of LD and HD phases in the form of a DW in CHI or CHII, so that with $n$ changing within the interval $n_{L}<n<n_{U}$ the total particle content in CHI and CHII changes due to shifts in the location of the LDW keeping $J_{I}$ and $J_{I I}$ unchanged.

With CHIII, having the lowest hopping rate being in its MC phase with $n_{3}=1 / 2$, using the continuity of current in each channel, we write

$$
\begin{array}{r}
n_{I}=\frac{1 \pm \sqrt{1-q}}{2}, \\
n_{I I}=\frac{1 \pm \sqrt{1-q / p}}{2} .
\end{array}
$$

The two solutions for each of $n_{I}$ and $n_{I I}$ represent either HD or LD phase densities for CHI and CHII, respectively. Since on physical ground, particles start accumulating behind the slowest segment (CHIII here), depending on the exact configuration of the model, an LDW will be formed either in the segment with hopping rate 1 or with hopping rate $p$. Thus, given Fig. 2, an LDW forms in CHII and then shifts towards CHI as more and more particles are added.

Consider now an LDW in CHII. As per our arguments, CHI will be in its LD phase given by $n_{I}=\frac{1-\sqrt{1-q}}{2}$. In order to maintain the same current $J_{I I}, n_{I I}$ will have two solutions which will meet at a point say, $x_{I I}^{w}$. Thus we have a discontinuous jump in the density profile at $x_{I I}^{w}\left(1 / 3<x_{I I}^{w}<2 / 3\right)$. As in the FSM [11], we identify effective particle injection rate, $\alpha_{2}=\frac{1-\sqrt{1-q / p}}{2}<1 / 2$ and an effective extraction rate, $\beta_{2}=\frac{1-\sqrt{1-q / p}}{2}<1 / 2$, thus satisfying the coexistence condition, $\alpha_{2}=\beta_{2}<1 / 2$.

Total particle number conservation in the model yields

$$
\left(\int_{0}^{1 / 3} n_{I}+\int_{1 / 3}^{2 / 3} n_{I I}+\int_{2 / 3}^{1} n_{I I I}\right) d x=n,
$$

The density profile, $n_{I I}$ with an LDW at $x_{I I}^{w}$ is given by $\alpha_{2}+\Theta\left(x-x_{I I}^{w}\right)\left(1-\alpha_{2}-\beta_{2}\right)$, where $\alpha_{2}=\beta_{2}=\frac{1-\sqrt{1-q / p}}{2}$. $\Theta$ is the Heaviside step function. The jump in the density by the value $1-\alpha_{2}-\beta_{2}$ at $x=x_{I I}^{w}$, gives the height of the DW.

The position of the LDW can be evaluated from Eq. 12. which gives

$$
x_{I I}^{w}=-\frac{n-1 / 2-\sqrt{1-q / p} / 2+\sqrt{1-q} / 6}{\sqrt{1-q / p}} .
$$

Similarly, if the DW is created in CHI, then CHII will be in its HD phase and the corresponding position for the LDW will be

$$
x_{I}^{w}=-\frac{n-1 / 2-(\sqrt{1-q / p}+\sqrt{1-q}) / 6}{\sqrt{1-q}} .
$$

The phase boundaries are obtained by setting $x_{I}^{w}=0$ and $x_{I I}^{w}=2 / 3$. For $x_{I}^{w}=0$, the equation for the boundary line reads

$$
n=n_{U}=1 / 2+(\sqrt{1-q / p}+\sqrt{1-q}) / 6,
$$

while for $x_{I I}^{w}=2 / 3$, we have

$$
n=n_{L}=1 / 2-(\sqrt{1-q / p}+\sqrt{1-q}) / 6 .
$$

We observe that the phase boundaries do not change if the transport direction was from CHI to Channel II via CHIII.

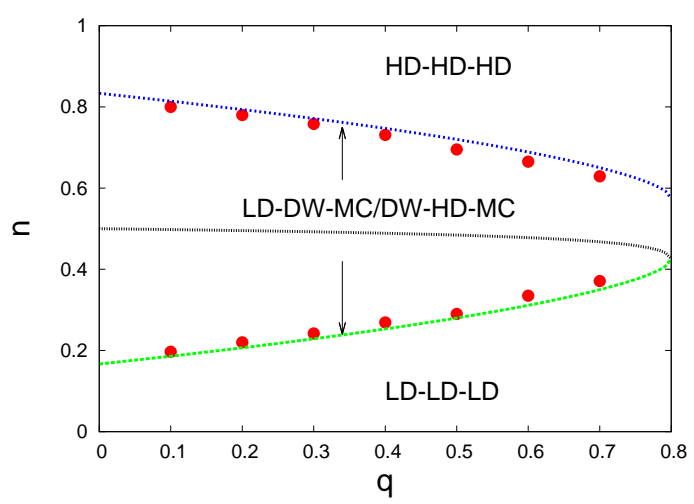

FIG. 3: Phase diagram of the 3-Channel system. Phase parameters are $n$ and $q$, with $p=0.8$. The black line represents the boundary when the LDW is formed at the junction of CHI and CHII. Points represent MCS results.

The phase diagram of the model in the $(n, q)$-plane, parametrized by $p$ is given in Fig. (3). Its qualitative nature may be discussed in simple physical terms. The DW-MC region shrinks as $q \rightarrow 0.8$. As $q$ decreases, the DW-MC region becomes wider having its maximum width as $q \rightarrow 0$. In this limit, CHIII has a vanishingly small hopping rate and hence the model is nearly blocked. Considering that the DW phase in CHI/CHII is accompanied by the MC phase in CHIII, thus CHIII must have 
a density $1 / 2$ to be in its MC phase. Notice that the current in the system in its DW-MC phase is $q / 4$ and hence decreases with decreasing $q$. Therefore, the total number of particles in CHI/CHII, required for them to reach an LD phase with a system current of $q / 4$ and hence, $n_{L}$ marking the threshold between the (LD-LD-LD) and (LD-DW-MC) phases, also comes down with $q$ accordingly. Using the particle-hole symmetry, we immediately conclude that the threshold $n_{U}$ between the (DW-HD$\mathrm{MC}$ ) and (HD-HD-HD) phase increases with decreasing $q$. This explains the increasing width of the (DW-MC) region for decreasing $q$ in the $(n, q)$ phase diagram. Taking density as the order parameter, we note that it changes continuously across the boundaries between the (LD-LDLD) and (LD-DW-MC) phases, and the (DW-HD-MC) and (HD-HD-HD) phases, corresponding to second order transitions. This is in contrast to the first order transition from the LD to HD phases in an open TASEP. Hence, the usual first order line in a TASEP is replaced by two second order lines here. Our MF results are complemented by extensive MC simulations, which were realised by using random sequential updates together with averages over large number of samples. Representative plots of $n_{I}(x), n_{I I}(x)$ and $n_{I I I}(x)(N=2100, p=0.8)$ in the (LD-LD-LD) and (LD-DW-MC) phases, obtained from both our MFT and MCS studies, are given in Fig. 4. Both figures (3) and (4) clearly show that while the MFT results match qualitatively with the corresponding MCS results, there are minor quantitative disagreements between them, presumably due to the correlation effects which are stronger in the closed model here than for an open TASEP. Notice that there can be only one LDW in the system, either in CHI or CHII. If it is in CHI (CHII), then CHII (CHI) will be entirely in its LD (HD) phase, characterised by uniform densities (neglecting BLs). Nonetheless, there will however be a jump in the density profile at the junction B with the jump height being controlled by the difference between the hopping rates in CHI and CHII respectively. This jump survives even in the thermodynamic limit. Thus, while in terms of the number of LDWs, our Model I yields the same results as the FSM model [11], Model I has additional density discontinuities in its steady states.

Clearly if we set $p=1$ in Model I, CHI and CHII become indistinguishable. In fact, with $p=1$, our Model I effectively reduces to the two-segment model in Ref. [11], such that the slower part has a length half of the faster part. At this limit, unsurprisingly our results reduce to those of Ref. [11]. A current vs density plot is shown in Fig. [5] corresponding to the LD-DW-MC phase in Fig. 4.

\section{MODEL II: FOUR SEGMENT MODEL}

We now extend our Model I to four-segment model (Model II), having four parts of equal number of sites, but with all unequal hopping rates along them (say, 1, $p_{1}, p_{2}, p_{3}$ with $\left.1 \geq p_{1} \geq p_{2} \geq p_{3}\right)$. Extending our argu-

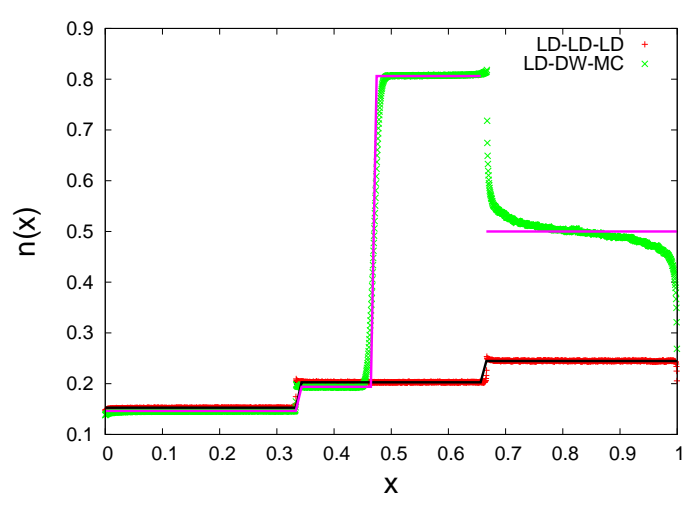

FIG. 4: Representative plots for (LD-LD-LD, $n=0.2, q=$ 0.7 ) and (LD-DW-MC, $n=0.4, q=0.5)$ phases with $p=0.8$ and $N=2100$. Points and solid lines represent MCS and MFT results, respectively.

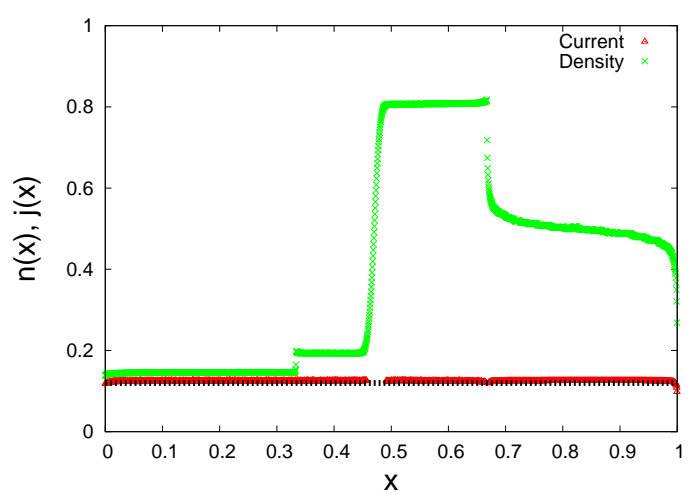

FIG. 5: Plot showing dependence of the steady state current in the bulk on the density $(p=0.8, q=0.5, n=0.4$ and $N=2100)$. The dashed black line represents the MFT result for the current while the points show MCS results. Since the MCS results for the steady state current is calculated from their mean field expressions, these are valid only in the bulk, away from the junctions and any discontinuity.

ments and analyses in the previous Section, it is clear that there are no qualitative changes in the overall density profiles of Model II, in comparison with Model I: Either all segments will be in their LD (or HD) phases, or, for intermediate densities, the segment with the lowest hopping rate $\left(p_{3}\right)$ reaches its MC phase, together with the simultaneous formation of an LDW in any of the other segments with its precise location being given by the total particle number conservation in the system. There are, however, surprises, when the different segments have hopping rates 1, $p, 1, p(1>p)$, respectively (see Fig. 6). Thus, CHI and CHIII are identical to each other, where as, CHII and CHIV are mutually identical.

Total particle number conservation and the equality of the current in the bulk of every segment allow us to calculate the density profiles in each segment, just as above. Let $n_{I}, n_{I I}, n_{I I I}, n_{I V}$ be the densities of CHI, CHII, CHIIII and CHIV, respectively. Physically, for very low 


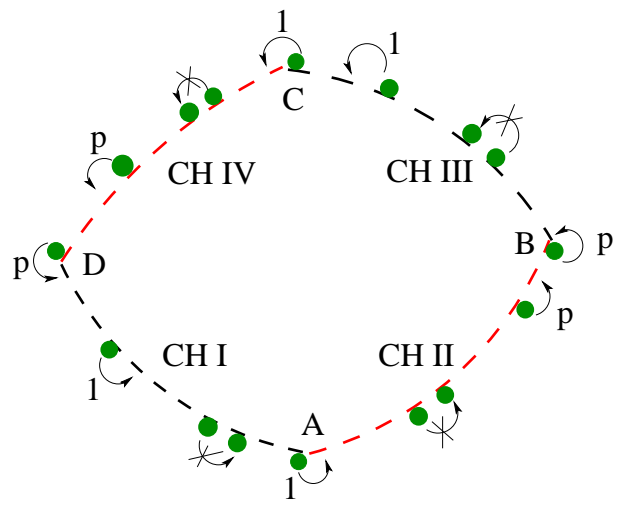

FIG. 6: Schematic diagram of the 4-sector model. CHI and CHIII (black) have a hopping rate $1, \mathrm{CHII}$ and $\mathrm{CH}$ IV (red) have a hopping rate of $p<1$ (see text).

(high) densities, all of CHI, CHII, CHIII, CHIV are in their LD (HD) phases, with $n_{I}=n_{I I I}$ and $n_{I I}=n_{I V}$ in the bulk. Proceeding as above, current conservation in the bulk yields

$n_{I}\left(1-n_{I}\right)=n_{I I I}\left(1-n_{I I I}\right)=p n_{I I}\left(1-n_{I I}\right)=p n_{I V}\left(1-n_{I V}\right)$,

allowing us to obtain $n_{I}=n_{I I I}$ in terms of $n_{I I}=n_{I V}$. Assuming $n_{I}+n_{I I}+n_{I I I}+n_{I V}=4 n, n$ being the total average density, we can thus write,

$$
n_{I}+n_{I I}=2 n
$$

This yields solutions for $n_{I}$ and $n_{I I}$, where,

$$
n_{I I}=\frac{-b \pm \sqrt{b^{2}-4 a c}}{2 a}
$$

with $a=(p-1) / 4, b=n-\frac{1+p}{4}$ and $c=n / 2-n^{2}$.

As more particles are added to the system, CHII and CHIV should reach their MC phases (similar to our analysis for Model I above). Then,

$$
n_{I}\left(1-n_{I}\right)=n_{I I I}\left(1-n_{I I I}\right)=p / 4,
$$

since the currents in CHII and CHIV in their MC phases are just $p / 4$. This allows us to solve for $n_{I}$ and $n_{I I I}$ :

$$
n_{I}=n_{I I I}=\frac{1 \pm \sqrt{1-p}}{2}
$$

Thus, for each of $n_{I}$ in CHI and $n_{I I I}$ in CHIII,we obtain two values, one greater and the other less than $1 / 2$. Following our physical picture for Model I, thus, an LDW should form connecting the two solutions for $n_{I}$ and $n_{I I I}$ at points in the bulk of CHI and CHIII, respectively.

Particle number conservation in the model yields

$$
\left(\int_{0}^{1 / 4} n_{I}+\int_{1 / 4}^{1 / 2} n_{I I}+\int_{1 / 2}^{3 / 4} n_{I I I}+\int_{3 / 4}^{1} n_{I V}\right) d x=n .
$$

We now define coordinates $0<x_{I}<1 / 4,1 / 4<x_{I I}<$ $1 / 2,1 / 2<x_{I I I}<3 / 4$ and $3 / 4<x_{I V}<1$ for CHI, CHII, CHIII and CHIV, respectively in order to find the location of the LDWs.

Let $x_{I w}$ and $x_{I I I w}$ be the locations of the LDWs in CHI and CHIII, respectively. The density profiles $n_{I}\left(x_{I w}\right)$ and $n_{I I I}\left(x_{I I I w}\right)$ may be written as

$$
n_{I}\left(x_{I w}\right)=\alpha+\Theta\left(x-x_{I w}\right)(1-\alpha-\beta)
$$

and

$$
n_{I I I}\left(x_{I I I w}\right)=\alpha+\Theta\left(x-x_{I I I w}\right)(1-\alpha-\beta),
$$

where, $\alpha=\beta=\frac{1-\sqrt{1-p}}{2}$.

Substituting for $n_{I}\left(x_{I} w\right)$ and $n_{I I I}\left(x_{I I I w}\right)$ and noting that $n_{I I}\left(x_{I I}\right)=1 / 2=n_{I V}\left(x_{I V}\right)$ in Eq. (22) (ignoring the boundary layers) we find

$$
\frac{1}{4}+\frac{\alpha}{2}+(1-2 \alpha)\left(1-x_{I w}-x_{I I I w}\right)=n .
$$

Hence, we have two unknown positions, $x_{I w}$ and $x_{I I I w}$, of the LDWs in CHI and CHIII, respectively, but only one equation (particle number conservation) that relates them. Therefore, $x_{I w}$ and $x_{I I I w}$ cannot be determined uniquely, resulting into delocalised domain walls (DDW) in each of CHI and CHIII. The fact that we obtain two DDWs instead of an LDW can be easily explained from the total particle number conservation. When there is a single LDW in the system, its position gets fixed by the particle number conservation. Evidently, particle number conservation can be maintained equally well for two LDWs by shifting one LDW in one direction and shifting the second LDW in the reverse direction by the same amount. Under long time averaging, the system displays an average of all the (pairwise) LDW positions satisfying total particle number conservation, yielding two DDWs. Our MFT results on DDWs are corroborated by our MCS studies on Model II; see Fig. 7 and Fig. 8 for DDWs in CHI and CHIII of Model II with two different system sizes. We present a kymograph (Fig. 91) depicting synchronisation of the movements of the DDWs in CHI and CHIII. The synchronised movements of the two DDWs are an outcome of the particle number conservation as discussed above.

\section{A. DDW profiles and the phase boundaries}

While $x_{I w}$ and $x_{I I I w}$ cannot be determined in MFT uniquely, their average profiles may be obtained. Noting that the steady state densities of CHI and CHIII should be statistically identical, due to the obvious symmetry between CHI and CHIII, we write $\left\langle n_{I}\left(x_{I}\right)\right\rangle=$ $\left\langle n_{I I I}\left(x_{I I I}\right)\right\rangle$, where $\langle\ldots\rangle$ represents configuration averages. Let us consider a position, $x_{0}$, in Channel I, where a DW has formed. Using the symmetry in the system, we can thus say that Channel III will have a DW 


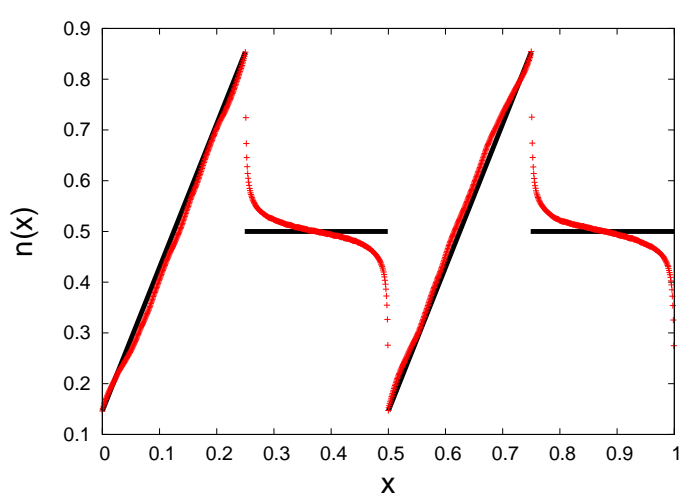

FIG. 7: Formation of DDWs for $N=1200, n=0.5, p=0.5$. The solid black lines and the red points represent MFT and MCS results, respectively. MCS results have been averaged over 10 samples of data. Simulation results show good agreement with the theory. Each sample was iterated for $2 \times 10^{9}$ steps.

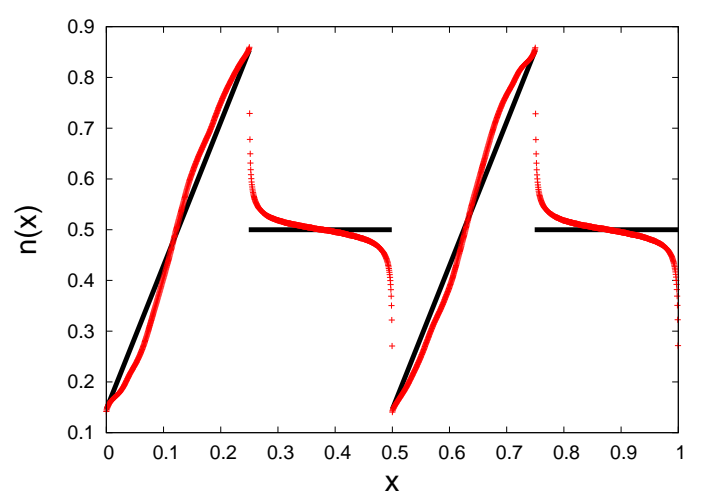

FIG. 8: Formation of DDWs for $N=2000, n=0.5, p=0.5$. The solid black lines and the red points represent MFT and MCS results, respectively. MCS results have been averaged for 32 different samples with each sample being iterated for $8 \times 10^{9}$ steps [23].

at a position, $\frac{1}{2}+x_{0}$. So on setting $\left\langle x_{1 w}\right\rangle=x_{0}$ and $\left\langle x_{I I I w}\right\rangle=\frac{1}{2}+x_{0}$, we obtain,

$$
x_{0}=-\frac{n-\frac{3}{4}+\frac{\alpha}{2}}{2(1-2 \alpha)}
$$

Clearly, the phase boundaries can be obtained by setting $x_{0}$ equal to 0 and $1 / 4$, respectively. This yields phase boundaries in the $(n, p)$ plane identical to those obtained for the equal-sized two-segment system (FSM), described in the beginning. Nonetheless, the underlying physical pictures are very different. In case of Model II, the phase boundaries mark the boundaries of the DDW-MC phases, where as, in case of FSM in Ref.[11], they refer to the boundaries of the LDW-MC region. While the topology remains invariant for the system (see Fig. 10), we find that in Model II the LDWs are not pinned to a particular position, resulting into formation of DDWs. Evidently, systems with presence of multiple slowest sectors cannot

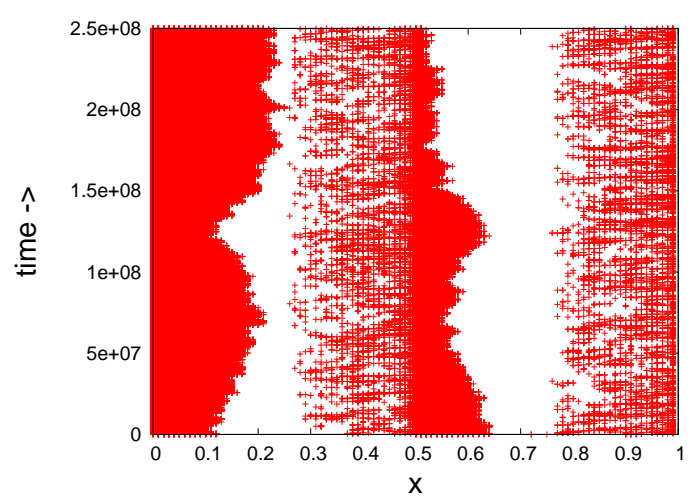

FIG. 9: Kymograph showing the synchronisation of Domain walls in CHI and CHIII. $N=800, n=0.5, p=0.5$ [24].

be reduced to an equivalent two-channel system. Representative plots of DDWs in CHI and CHIII, as obtained from both MFT and MCS studies, are shown in Fig. 7 and Fig. 8, Good agreement between our MCS are MFT results are visible.

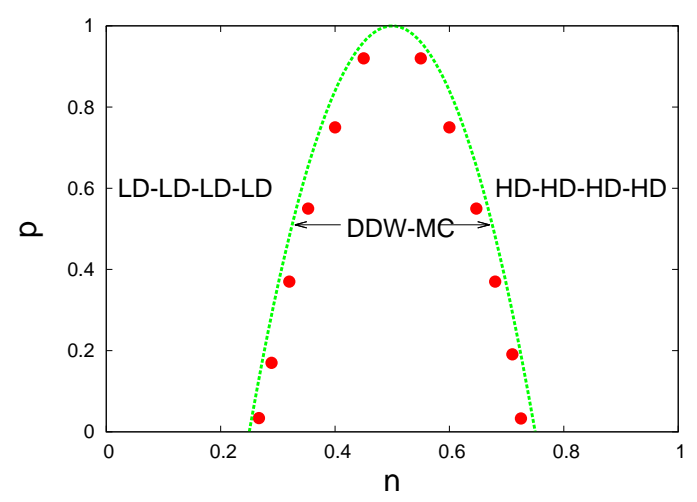

FIG. 10: Phase diagram of the four segment model. The domain walls formed are delocalised in contrast to the two and three segment systems. Points and lines represent MCS and MFT results, respectively.

\section{POSSIBLE DENSITY PROFILES FOR ARBITRARY NUMBER OF SEGMENTS WITH UNEQUAL HOPPING RATES}

Now we generalise our system to arbitrary number of segments with unequal hopping rates. For such cases, our results above for three and four segments may be generalised to obtain information about the steady state density profiles, which we briefly outline here. For instance, consider an $n$-segment system with hopping rates $1>q_{2}>q_{3}, \ldots, q_{n}$, respectively of the segments marked, $1,2, \ldots, n$. Thus, the $n$th segment is the slowest segment. Now, based on our arguments elaborated above, we can conclude that the $n$th segment should reach its MC phase for intermediate densities. At the same time, an LDW 
will be formed in any one of the other remaining segments, whose position is controlled by the overall particle number density. All the remaining faster segments are to have uniform bulk densities. Nevertheless, as in our Model I, there should be additional discontinuities in the density profiles at the junctions of the different faster segments (i.e, at the junctions between the segments $1,2, \ldots, n-1)$. Thus, we generally conclude that for any number of finite inhomogeneous segments, all having unequal hopping rates, the slowest segment will reach its MC phase for intermediate densities. At the same time, an LDW will be formed in any one of the other remaining segments, whose position may be obtained by the overall particle number density. Consider now the case where two of the nonconsequtive segments have the slowest hopping rates. For concreteness, assume in the system $q_{p}=q_{s}$ are the lowest hopping rates associated with segments $p$ and $s$, respectively; $p, s$ not being neighbours of each other. This would be a generalisation of our Model II above. In this case, again based on our arguments above, we argue that for intermediate densities two DDWs should form behind each of the slowest segments ( $p$ th and $s$ th, in the case). In fact, if there are several disjoint slowest segments, we expect those many DDWs to form behind each of the slowest segments. Calculation of the DDW profiles remains a non-trivial task. In the simplest case, the DDW profiles may be obtained by exploiting the structural symmetry of the system (as in our Model II). Overall, then, for intermediate densities, the system should either have a combination of an MC phase (in the slowest segment) and one LDW elsewhere in one of the faster segments, or, a combination of several MC phases (when there are more than one nonconsecutive slowest segments) and an equal number of DDWs behind each of the slowest segments. Of course, for very low or very high densities, all the segments should be in either LD or HD phase, regardless of the details of the hopping rates of the individual segments.

\section{FLUCTUATIONS OF DOMAIN WALLS}

\section{A. Fluctuations of LDWs}

In our MF description of Model I, where all fluctuations are neglected, an LDW is assumed to be static at $x_{I}^{w}$. In contrast, our MCS reveal that an LDW, say in CHI, fluctuates around its mean position $x_{I}^{w}$, which MFT cannot capture. Since there are no qualitative distinctions between the LDWs in the FSM model of Ref. [1] and our Model I, we use the FSM model to illustrate the fluctuations of the domain walls for simplicity. We characterise the LDW fluctuations numerically here by means of our MCS studies. We study the fluctuations for two different sizes of the defect regions. The size of the defect region relative to the total system size is characterised by $l$; see Fig[11, $l=1 / 2$ represents the case where the defects cover half of the system size and $l=0.25$ represents

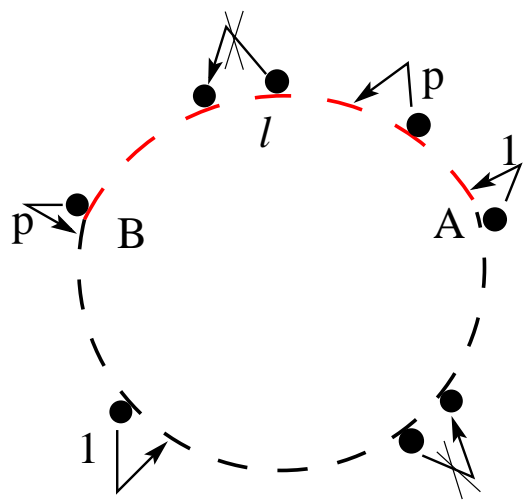

FIG. 11: Two segment model (FSM) with unequal sizes of the two segments. The relative length of the defect region is captured by $l ; l=1 / 2$ represents equal sizes of the two segments (see text).

$1 / 4$ th of the system being covered with defects. The width, $\sigma$ of the distribution of DW fluctuations can be obtained by fitting the density profile in the vicinity of the domain wall by the function $(P \cdot \operatorname{erf}[(x-Q) / \sigma]+R)$ [25], with the parameters $P, Q, R, \sigma$. Our studies reveal normalized width $\sigma(N) / N \sim 1 / \sqrt{N}$ (within error bars) for both $n \neq 1 / 2$ and $n=1 / 2$. For $l=1 / 2, n=0.5$, we have used $N=800,1600,2400,3000,4000$ and for $l=1 / 2, n=0.6, N=800,1600,2400,3200,4000$. For $l=0.25$ and $n=0.5$, we have checked with system sizes $800,1200,1600,2000,2400$ and while $l=0.25$ and $n=0.6$, we have used $N=1200,1600,2400,3200,4000$; see Fig. 12.

Recall now the studies in Ref. [12] on a ring TASEP with a point bottleneck, where $\sigma / N$ is shown to scale as $1 / \sqrt{N}$ for $n \neq 1 / 2$, where as $\sigma / N \sim 1 / N^{2 / 3}$ for $n=1 / 2$. The different scaling law for $n=1 / 2$ is ascribed to a special symmetry at $n=1 / 2$ by which particles and holes, created pairwise at the bottleneck, impinge upon the DW pairwise 12. In contrast, the FSM model of Ref. 11] does not have any special symmetry for $n=1 / 2$. This is due to the extended size of CHII (i.e., an extended defect unlike a point defect in the model of Ref. [12]), for which particles and holes created at the boundaries between CHI and CHII do not impinge upon the DW for $n=1 / 2$ pairwise; in addition, careful observation of the density profiles obtained in our MCS studies reveal additional BLs in Model I, (see Fig. (4), a consequence of correlations borne out of strict particle conservations. Similar BLs are found in our MCS studies of the two-segment model (FSM) as well (not shown here). These BLs appear to survive for all $N$ in our MCS. We expect these BLs should invalidate the special symmetry at $n=1 / 2$ of Ref. 12] further in the present context. These factors should be responsible for our difference in the scaling of $\sigma(N)$ with Ref. [12] for $n=1 / 2$. This $1 / \sqrt{N}$ scaling for the width $\sigma(N) / N$ may be understood heuristically as follows. Due to the stochastic nature of the microscopic dynamics, the particle current past a given point 


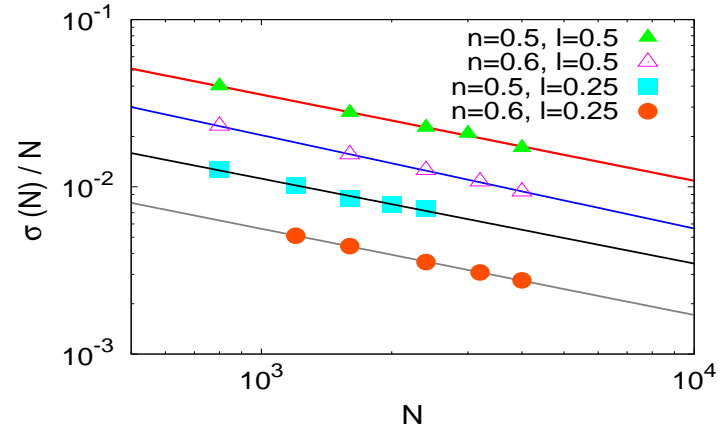

FIG. 12: Log-log plot of the normalised LDW widths $\sigma(N) / N$ versus $N: \sigma(N) / N \sim 1 / \sqrt{N}$ within error bars.(Different data sets are shifted vertically for better visual clarity.)

in the system is not static even in the steady state; it fluctuates randomly about its mean value as given by MFT. As a result, the number of particles entering (exiting) the segment with an LDW (i.e., the segment with a higher hopping rate) fluctuates randomly around their mean values. These fluctuations $\delta N_{p}$ lead to the fluctuations in the LDW position, measured by $\sigma$. Let $N_{p}$ be the average particle number in that segment. In a $1 \mathrm{D}$ system with exclusion as ours, it is reasonable to assume $\sigma \propto \delta N_{p}$. Assuming no long-ranged correlations, we write $\delta N_{p} / N_{p} \sim 1 / \sqrt{N}_{p}$. Since $N_{p}$ should scale with $N$ for a given density and inhomogeneity configuration, we find $\sigma(N) / N \sim 1 / \sqrt{N}$. In the absence of any special symmetry at $n=1 / 2$, we expect the above simple picture should hold good even for $n=1 / 2$.

\section{B. Delocalisation transition and fluctuation of DDWs}

As we have seen before, in our Model II with hopping rates $1, p, 1, p$ in CHI, CHII, CHIII, CHIV, respectively, the densities in CHI and CHIII display DDWs for moderate densities, when CHII and CHIV are in their MC phases. On the other hand, if CHII and CHIV do not have same hopping rates $p$, there will be only one LDW in the system at a location governed by the particle number conservation. Therefore, as the hopping rates in CHII and CHIV approach each other, this LDW should undergo a delocalisation transition and two DDWs emerge in CHI and CHIII. Thus, the width of the LDW fluctuation should diverge in the thermodynamic limit at the delocalisation transition. We investigate this by MCS studies of our Model II.

In Fig. 6, assume CHII and CHIV have hopping rates of $p-\epsilon$ and $p+\epsilon$, respectively. Hopping rates at CHI and CHIII continues to be unity (as before). Consider $n$ to be such that CHII be in its MC phase. As $\epsilon \rightarrow 0$, an MC phase should be formed in CHIV and the LDW should approach a delocalisation transition, i.e., the width of the LDW is expected to diverge as $\epsilon \rightarrow 0$. We investigate how the width of the LDW, $\sigma$ scales with $\epsilon$ by fitting the density profile in the vicinity of the domain wall by the function $(P \cdot \operatorname{erf}[(x-Q) / \sigma]+R)$, with the parameters $P, Q, R, \sigma$. (as above), for a given $N$. MCS studies reveal that, $\sigma \propto \frac{1}{\epsilon^{1 / 2}}$ (within error bars; see Fig 13 ), diverging as $\epsilon \rightarrow 0$ (or, the normalized width $\sigma(N) / N$ is a finite value in the limit $\epsilon \rightarrow 0$ in the thermodynamic limit $N \rightarrow \infty$ ). This establishes scaling of the domain wall positions near the delocalisation transition in our Model II.

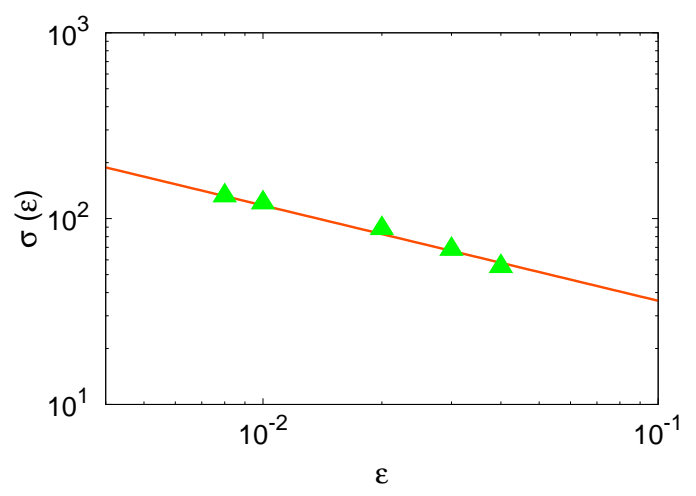

FIG. 13: Log-log plot for the scaling of the width, $\sigma$ with $\epsilon$ for the 4-segment system $(N=800, n=0.44) ; \sigma \propto \epsilon^{-1 / 2}$.

A rigorous mathematical formulation of the scaling law $\sigma \propto \frac{1}{\epsilon^{1 / 2}}$ may possibly be done by using the formalism developed in Ref. [26] appropriately modified for a closed system. We do not do that here; however, we make interesting analogies of the delocalisation transitions discussed above with second order phase transitions in (equilibrium) magnetic spin systems. Considering the scale of the domain wall fluctuations as a correlation length $\xi$ of density fluctuations (since this sets the scale of the fluctuations of domain wall positions), we have $\xi \propto \frac{1}{\epsilon^{1 / 2}}$. This scaling behaviour is reminiscent of the correlation length $\sim 1 / \sqrt{T-T_{c}}$ in a magnetic system very close to the critical temperature $T_{c}$. Qualitatively, for a magnetic system at $T_{c}$, the free energy potential well becomes flat even for large values of the magnetisation, leading to large magnetisation fluctuations. Similarly in the present model, the positions of the underlying LDWs of a DDW envelope can be anywhere within the DDW envelope with equal probability. Further theoretical investigations going beyond MFT should be useful in studying this analogy [27].

\section{CONCLUSION}

In this work, we analyse the generic steady state density profiles in TASEP on a ring with extended inhomogeneities. We find for moderate average densities, nontrivial spatial dependences for the density profiles in the steady states, including LDW, along with second order transitions, and localisation-delocalisation transition 
leading to DDWs. The underlying inhomogeneity configuration together with the overall particle number density controls the macroscopic density profiles. For instance, we demonstrate that for very low (high) densities, the system is in homogeneous LD (HD) phases, regardless of the inhomogeneity configuration (i.e., for both Model I and Model II above). For moderate densities, the underlying inhomogeneity configurations become crucial. We find that in our Model I, having three segments with unequal hopping rates, the overall behavior is qualitatively similar to that of the FSM model of Ref. [11], having two segments of unequal hopping rates. Both Model I and FSM display one LDW for moderate densities, the location of which is determined essentially by the particle number conservation. When the number of segments increases to four (Model II), with all unequal hopping rates, again there is generically only one LDW for intermediate densities, similar to our Model I. However, there are now additional density discontinuities at the junctions between the faster segments. Surprisingly, in Model II for specially constructed inhomogeneity configurations with hopping rates $1, p, 1, p ; p<1$, the macroscopic density profiles are significantly different in having two DDWs, instead of one LDW. We argue that this is due to the existence of more than one (two here) slowest segments in Model II. In general, our MFT and MCS results match qualitatively for both Model I and Model II, in general. While the instantaneous DDW positions cannot be determined cannot be determined from MFT, we discuss how their long-time averaged density profiles may be constructed by using MFT. We use our detailed results from Model I and Model II to infer about the possible steady state density profiles and argue that depending upon whether the system has one or more than one slowest segments, either the system should show an LDW or a number of DDWs (equal to the number of the slowest segments). Notice that the current-density relationship in a given segment is formally continued to be given by the same expression as for an isolated open TASEP with an arbitrary hopping rate. Equality of the steady state currents in all the segments ensures that in the slowest segment(s), the current can reach its maximum value corresponding to the $\mathrm{MC}$ phase(s). Thus the form of the current versus density plots for the slowest segments should be identical in nature to that for an open TASEP. In contrast, the maximum currents in the faster segments will be limited by the extended defects (the slowest segments), as these segments will not reach their MC phases. Thus, the currents in these faster segments will be less than their values in their putative MC phases had they been isolated open TASEPs. Hence, in the corresponding current-density plots, the maximum value of the current will be truncated to a value controlled by the $\mathrm{MC}$ phase currents in the slowest segments, see for example [11], 13]. Lastly, we study the fluctuations of LDW and DDWs numerically. We show that the relative width of an LDW scales as $1 / \sqrt{N}$, such that in the thermodynamic limit $N \rightarrow \infty$, an LDW becomes fully sharp. We also numerically illustrate the delocalisation transitions of an LDW in our Model II, when the hopping rates of two of the slower segments approach each other. Assuming hopping rates of the four segments in Model II as $1, p+\epsilon, 1, p-\epsilon$, two DDWs are formed, with their spans diverging in the thermodynamic limit $N \rightarrow \infty$ for $\epsilon=0$. We numerically establish that as $\epsilon \rightarrow 0$, the width, $\sigma \propto 1 / \sqrt{\epsilon}[28]$. Overall, at the broader level our work reveals how the mutual interplay between the particle number conservation and the quenched disorder configurations yield different types of density profiles including LDW and DDWs.

Our model serves as a simple starting point to study the effects of an arbitrary number of extended inhomogeneities on asymmetric exclusion processes in a closed system. Additional biologically relevant details may be incorporated in our model, e.g., the detailed features of the model discussed in Ref. [29]. On the theoretical side, how nonconservation of particles may affect the density profiles obtained here should be a worthwhile question to study. Due to the minimalist nature of the model, we do not expect immediate quantitative agreement with specific experimental results. Nevertheless, qualitative features of our results may be tested in ribosome density mapping [30] or ribosome profiling [31] experiments on model mRNA loops with clusters of slow codons. In particular, it may be noted that arguments those yield the existence of LDW or DDWs in our models for intermediate densities are independent of the precise lengths of the extended defects. Thus, while we have assumed equal lengths for the segments in both Model I and Model II for simplicity, we expect the qualitative features of LDW and DDW should survive even for short segments of several extended defects. This may be of particular interests in the context of mRNA loops with slow codons, where the latter generally occur in several short segments. In the context of vehicular traffic flows, our model shows under what condition there may be crowding of vehicles behind extended bottlenecks in a closed network of roads. Therefore, it is expected that in a closed network of narrow roads with no possibility of overtaking, one would generically observe either moving jams (DDWs) or static jams (LDW), depending upon the geometry of the defects. In addition, possible experimental realisations on the unidirectional restricted motion of interacting particles in narrow micropores (with the mutual passage excluded), e.g., in closed channels with circular geometry with extended bottlenecks [32] should make our model an intriguing system to study.

a. Acknowledgement:- We thank the anonymous Referees for their constructive suggestions in course of the review process. One of the authors (AB) wishes to thank the Max-Planck-Gesellschaft (Germany) and the Department of Science and Technology/Indo-German Science and Technology Centre (India) for partial financial support through the Partner Group programme (2009). 
[1] R. K. P. Zia, J. J. Dong and B. Schmittmann, J. Stat. Phys. 144, 405 (2011).

[2] J. Kärger and D. Ruthven, Diffusion in zeolites and other microporous solids (Wiley, New York, 1992).

[3] H J Hilhorst and C Appert-Rolland, J. Stat. Mech.: Th. Exp. P06009 (2012).

[4] T. Chou, K. Mallick and R. K. P. Zia, Rep. Prog. Phys. 74, 116601 (2011).

[5] A. Parmeggiani, T. Franosch and E. Frey, Phys. Rev. Lett. 90, 086601 (2003).

[6] I. Neri, N. Kern and A. Parmeggiani, Phys. Rev. Lett. 110, 098102 (2013);I. Neri, N. Kern and A. Parmeggiani, Phys. Rev. Lett. 107, 068702 (2011)

[7] A. B. Kolomeisky, J. Phys. A 31, 1153 (1998); M. Liu et al, Phys. Lett. A 373, 195 (2009).

[8] M. Liu et al, Phys. Lett. A 374, 1407 (2010).

[9] P. C. Hohenberg and B. I. Halperin, Rev. Mod. Phys. 49, 435 (1977).

[10] H. Hinsch and E. Frey, Phys. Rev. Lett. 97, 095701 (2006); R. Chatterjee, A. K. Chandra and A. Basu, Phys. Rev. E 87, 032157 (2013); Niladri Sarkar and Abhik Basu, Phys. Rev. E 90, 022109.

[11] G. Tripathy and M. Barma, Phys. Rev. E 58, 1911 (1998)

[12] S. A. Janowsky and J. L. Lebowitz, Phys. Rev. A 45, 618 (1992).

[13] R. J. Harris and R. B. Stinchcombe, Phys. Rev. E 70, 016108 (2004).

[14] J. Szavits-Nossan, J. Phys. A 46315001 (2013).

[15] S.L.A. de Queiroz and R. B. Stinchcombe, Phys. Rev. E 78, 031106 (2008).

[16] M. Kardar, G. Parisi, and Y.-C. Zhang, Phys. Rev. Lett. 56, 889 (1986).

[17] J. Krug, Phys. Rev. Lett. 67, 1882 (1991).

[18] Under certain conditions mRNA strands may form closed loops facilitating ribosome recycling, see, e.g., T. Chou, Biophys. J. 85, 755 (2003); S. E. Wells, E. Hillner, R. D. Vale and A. B. Sachs, Mol. Cell. 2, 135 (1998); S. Wang,
K. S. Browning and W. A. Miller, EMBO J. 16, 4107 (1997).

[19] G. S. Kopeina et al, Nucleic Acids Res., 36, 2476 (2008).

[20] M. Robinson et al, Nucleic Acids Res. 12, 6663 (1984).

[21] M. A. Sorensen, C. G. Kurland and S. Pedersen, J. Mol. Biol. 207, 365 (1989).

[22] D. A. Phoenix and E. Korotkov, FEMS Microbiol. Lett. 155, 63 (1997).

[23] Much longer time averages would remove the minor disagreement between the MFT and MCS results in Fig. 8

[24] The mesoscopic length, say $r$, used to draw a kymograph, i.e., the length over which the spatial averaging is done should be much smaller than N. We have used $r=6$.

[25] T. Reichenbach, T. Franosch and E. Frey, Phys. Rev. Lett. 97, 050603 (2006).

[26] T. Reichenbach, T. Franosch and E. Frey, Eur. Phys. J. E 27, 47 (2008)

[27] It will be interesting to see how strictly the analogy between delocalisation transitions here and phase transitions in equilibrium magnetic systems hold due to the inherent nonequilibrium nature of the dynamics in the present model.

[28] For a finite system, this divergence will be cut off by the finiteness of the system. This is analogous to rounding of the critical divergenges at the critical temperature in finite systems. In the theoretical $N \rightarrow \infty$ limit, the scaling law $\sigma \propto 1 / \sqrt{\epsilon}$ should be observed without any rounding effect in the theromodynamic limit.

[29] A K Sharma and D Chowdhury, J. Theo. Biol. 289, 36 (2011), and references therein.

[30] Y. Arava et al, Nucl. Acids Res. 33, 2421 (2005).

[31] N. T. Ingolia et al, Science 324, 218 (2009); H. Guo et al, Nature 466, 835 (2010).

[32] Q.-H. Wei, C. Bechinger and P. Leiderer, Science 287, $625(2000)$. 